\title{
Direct Stereo Visual Odometry Based on Lines
}

\author{
Thomas Holzmann, Friedrich Fraundorfer and Horst Bischof \\ Institute for Computer Graphics and Vision \\ Graz University of Technology, Austria \\ \{holzmann, fraundorfer, bischof\}@icg.tugraz.at
}

\begin{abstract}
Keywords: Visual Odometry, Pose Estimation, Simultaneous Localisation And Mapping
Abstract: We propose a novel stereo visual odometry approach, which is especially suited for poorly textured environments. We introduce a novel, fast line segment detector and matcher, which detects vertical lines supported by an IMU. The patches around lines are then used to directly estimate the pose of consecutive cameras by minimizing the photometric error. Our algorithm outperforms state-of-the-art approaches in challenging environments. Our implementation runs in real-time and is therefore well suited for various robotics and augmented reality applications.
\end{abstract}

\section{INTRODUCTION}

In the last years, Simultaneous Localization and Mapping (SLAM) and Visual Odometry (VO) became an increasingly popular field of research. In comparison to Structure from Motion (SfM), which is not constrained to run in realtime, SLAM and VO methods should be able to run in realtime even on lightweight mobile computing solutions.

Visual localization and mapping approaches are often used for small robotics devices (e.g. Micro Aerial Vehicles (MAVs)), where it is not possible to use heavier and more energy-consuming sensors like laser scanners. However, most of these devices are equipped with an Inertial Measurement Unit (IMU), which can be used in conjunction with vision methods to improve the results.

There exist widely used monocular SLAM/VO methods, which just use one camera as the only information source and therefore can be used on many different mobile devices. In contrast to monocular approaches, stereo VO methods using a stereo setup with a fixed baseline have some benefits, especially when used for Robotics: (1) Monocular approaches cannot correctly determine the metric scale of a scene, while stereo approaches do have a correct scale as the baseline of the camera setup is fixed. Additionally, monocular approaches also have a drift in scale. (2) Monocular approaches do have problems with pure rotations as no new 3D scene information can be computed from images without translational movement. Contrary, stereo approaches do not have this problems, as a stereo rig already contains translationally moved cameras to triangulate scene information regardless of the motion of the cameras.

Based on the observation that man-made scenes usually contain strong line structures, like, e.g, doors or windows on white walls, we especially want to use these structures in our approach. Current featurepoint based methods (e.g. (Geiger et al., 2011)) have problems in these scenes, as the lack of texture leads to a low number of detected feature points. Direct pose estimation methods (e.g. (Engel et al., 2014)) directly use the image information to align consecutive frames and do not use explicitly detected feature point correspondences to estimate the camera pose. Therefore, direct methods perform better in poorly textured environments, as they use more image information and do not rely on detected feature points in the scene.

In this paper, we present a pose estimation method specifically targeted at poorly textured indoor scenes where other methods deliver poor results. We estimate the pose of a camera by minimizing the photometric error of patches around lines. We introduce a fast line detector which detects vertical lines aided by an inertial measurement unit (IMU). In case too few lines could be detected, we additionally detect point features and use patches around the detected points for direct pose estimation. In our experiments we show that our method outperforms state-of-the-art monocular and stereo approaches in poorly textured indoor scenes and delivers comparable results on well textured outdoor scenes. 
To summarize, our main contributions are:

- A direct stereo VO method using lines running in real-time.

- A novel efficient line detection algorithm aided by an IMU, that detects vertical lines.

- A fast line matching technique using a lightweight line descriptor.

\section{RELATED WORK}

A widely used approach to estimate the pose of a camera using the image data only is to use feature points in images. Feature points are detected and matched between subsequent images and used to estimate the pose of the camera. A popular monocular point-based SLAM-approach is PTAM (Klein and Murray, 2007). It runs in real-time and is specifically designed to track a hand-held camera in a small augmented reality workspace. However, there also exist adoptions tailored at large-scale environments ((Mei et al., 2010), (Weiss et al., 2013)). As it needs to detect and match feature points, the environment has to contain sufficient texture suited for the feature point detection algorithm. Additionally, as it is a monocular approach, it has difficulties in handling pure rotations and cannot estimate a correct scale of the scene. A feature-based approach, which uses a stereo camera as input and therefore does not have this problems is Libviso (Geiger et al., 2011). However, as it also uses explicitly detected feature points, the texture needs to be adequate for the detection algorithm. Additionally, as it is not a SLAM method like PTAM but just a VO method, it does not compute a global map of the environment but just computes the relative pose from frame to frame.

Instead of using points, also lines can be detected and matched in order to compute the pose of a camera. In (Elqursh and Elgammal, 2011), Elqursh et al. propose to estimate the relative pose of two cameras by using three lines having a special primitive configuration. This has the advantage that no texture at all needs to be present, but just a special line configuration has to exist. However, as explicit line detection is relatively slow, this approach cannot be used on a low-end onboard computer.

Recently, direct pose estimation algorithms became popular. In comparison to feature point-based approaches, direct approaches do not explicitly detect and match features and compute the pose using the feature matches, but compute the new pose by minimizing the photometric error over the whole or over big parts of the image. For example, DTAM (New- combe et al., 2011) tracks the pose of a monocular camera given a dense model of the scene, by minimizing the photometric error of the current image according to the whole model. As this is a computational expensive task, a high-performance GPU is necessary to compute the pose in real-time. Similarly, LSDSLAM (Engel et al., 2014) computes the pose by minimizing the photometric error. However, to reduce computational cost, it just uses areas in the image where the image gradient is sufficiently high. Therefore, it runs in real-time even on hand-held devices like smartphones. Also an extension of LSD-SLAM to a stereo setup has been proposed recently (Engel et al., 2015). In (Forster et al., 2014), an approach is proposed which uses both explicit feature point matching and direct alignment and is therefore called semi-direct visual odometry (SVO). It detects feature points at keyframes and computes the poses of images between keyframes by minimizing the photometric error of patches around the feature points. It runs very fast even on onboard computers and is specifically designed for a downwards looking camera of a micro aerial vehicle. In summary, direct methods perform direct image alignment to estimate the pose of a new frame with respect to previously computed $3 \mathrm{D}$ information by optimizing the pose parameters directly and by minimizing a photometric error. This is in contrast to feature-based methods, where features are detected and matched to compute the fundamental matrix, and optimization is just optionally used to refine the computed poses by using the reprojection error.

Our method is a direct method, but utilizes a stereo camera rig instead of a monocular camera. In contrast to all previous related work, we detect lines and estimate the pose by minimizing the photometric error of patches around the lines. Using our line detector, it is possible to detect lines very fast in constrained images. As in man-made environments most of the structure consists of lines, using patches around lines is usually sufficient to directly align consecutive images.

\section{DIRECT VISUAL ODOMETRY BASED ON VERTICAL LINES}

In our approach, we explicitly use the structures contained in man-made environments to introduce a novel visual odometry approach.

Instead of directly aligning the whole image or explicitly detecting and matching feature points, we estimate the camera pose by aligning patches just around detected lines by minimizing the photometric 


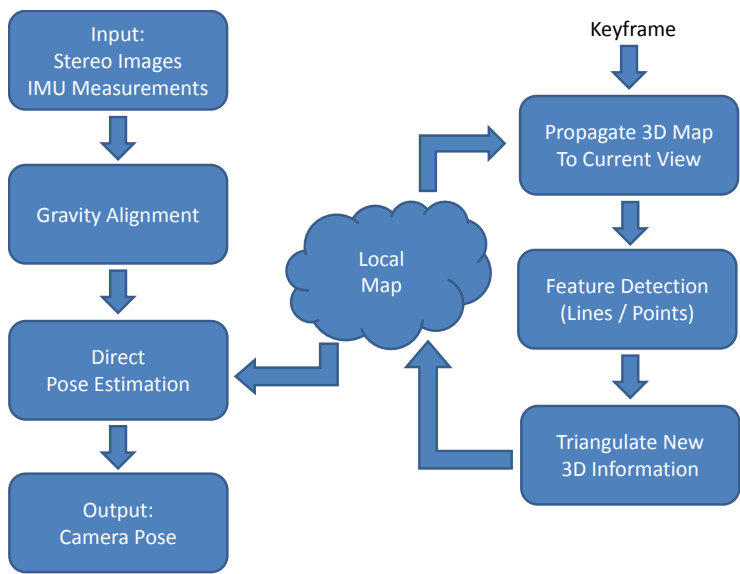

Figure 1: Overview of the processing pipeline. Our algorithm directly estimates the pose of the camera by minimizing the photometric error of patches around vertical lines. For every keyframe, the 3D information from the previous keyframe gets projected into the current one, new lines and optionally points get detected and new 3D information is triangulated within the stereo setup.

error. In case no lines can be detected, feature points are detected and patches around the points are used for image alignment.

\subsection{System Overview}

Our approach is a keyframe-based stereo approach, which takes rectified stereo images and optionally synchronized IMU values as input. As preprocessing, we align the images according to the gravity direction using the IMU measurements. Without IMU measurements, our approach also works on images acquired with roughly gravity aligned cameras.

For every keyframe, we detect and match lines using gravity-aligned stereo images. If not enough lines can be matched, we additionally detect and match point features. We triangulate this information to get a mapping of the visible information in 3D. A new keyframe is selected when the photometric properties of the images changed too much or the movement w.r.t the last keyframe is too big.

For every image, the pose gets estimated by direct image alignment: We project all triangulated information of the previous keyframe into the current image and estimate the pose by minimizing the photometric error of patches around the lines and points.

Additionally, for every consecutive keyframe, we project lines and points from the previous one into the current one to reuse already computed 3D information if the same lines or points are detected.

In Fig. 1, one can see a schematic overview of our processing pipeline.


Figure 2: Image alignment according to the gravity direction. Left: An unaligned image from a forwards looking camera Right: Image aligned using the IMU. As one can see, having an image aligned according to the gravity direction, straight, vertical lines in the 3D scene are mapped to straight, vertical lines in the image.

\subsection{Fast Line Detection and Matching}

Areas around lines contain high gradient values which is an important property for direct image alignment. Therefore, we introduce a fast line detection and matching method, where the area around lines is used for direct image alignment afterwards. We compute the depth of the lines by computing the disparity of the lines using the stereo image pair.

\subsubsection{Line Detection}

Usually, line detection (like, e.g. (Grompone et al., 2010)) is too slow for real-time applications (Hofer et al., 2014). To make the problem feasible, we focus on the detection of vertical lines, which can be done much faster. Man-made environments usually contain many vertical lines. Especially indoors, where the texture available very often mainly consists of lines, it is crucial for a visual odometry approach to use also this information. We introduce a detection algorithm aided by IMU information to make this challenging line detection step feasible: Having cameras with arbitrary poses and synchronized IMU information as input, the images can be aligned with the gravity direction using the simultaneously acquired IMU data (see Fig. 2). As the angular accuracy of IMUs is quite high (e.g., evaluation of the IMU of the Pixhawk autopilot (Cortinovis, 2010)) and most of the mobile computing devices already contain built-in IMUs, this preprocessing step can be done with many available mobile computing devices.

Even if the alignment is not completely perfect, it does not influence the pose estimation step, as the lines are not directly used for pose estimation but instead the patches around them. Errors in the alignment just result in a poorer line detection performance which can lead to shorter detected lines.

Assuming gravity aligned images as input, the task of detecting vertical lines gets easier as realworld vertical lines are now projected to vertical lines 


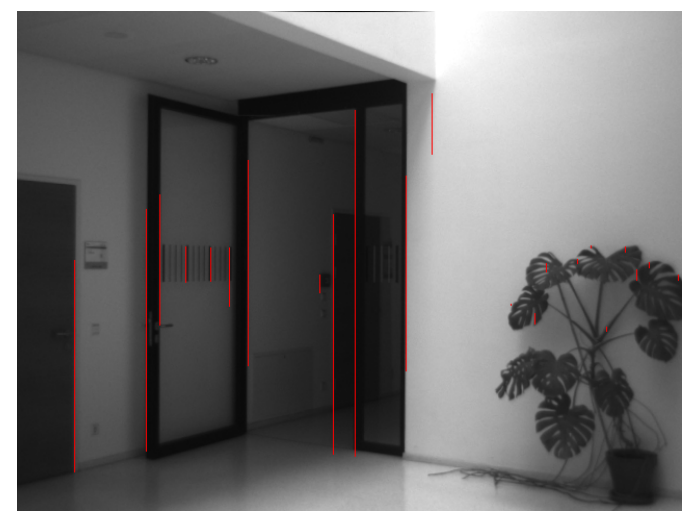

Figure 3: Vertical lines detected with our line detection algorithm. One can see that the lines are not detected over the whole length all the time. This is due to slight gravity misalignment of the images or due to changing light conditions.

in the image. Using this assumption, our algorithm first computes the image gradient in horizontal direction by convolving the image with the Sobel operator. Next, for every column of the image, a histogram of the gradient values is created. When a histogram bin associated with sufficiently high gradient values contains enough elements, a line is detected in this column.

As the lines are detected with pixel accuracy and, therefore, the number of lines is limited by the number of columns, we have a small and limited number of lines which increases the speed of the following line matching step.

After the detection of lines, we apply a simple non-maxima suppression: Lines get accepted, if their corresponding gradient histogram bin contains more elements than every gradient histogram bin corresponding to nearby lines.

Finally, the start and end point of the line is detected. In the gradient image, we search along the associated column for the first pixel belonging to the same bin as the corresponding line bin. This defines the start point of the line. Next, we search for a pixel belonging to a different bin. This defines the end point of the line. In order to be robust against noise and short discontinuities of the line, pixels with a similar histogram bin and discontinuities of a few pixels are also assigned to the line. Fig. 3 shows a result of the line detection.

\subsubsection{Line Matching}

We iterate over all lines in the left image and try to find corresponding lines in the right image. Between a predefined minimum and maximum disparity, we store all lines as potential matches which were de- tected having a similar gradient histogram bin as the left line. Then, we compute the Sum of Absolute Differences (SAD) of a patch around the line in the left image and the potential line matches in the right image.

For this, we need a equally-sized patch around the line in the left and right image. However, as the epipolar constraints do not hold anymore for the gravity aligned images (the images have been rotated after rectification), it is not possible to use just the maximum and minimum y-positions of the lines, as it would be possible in the original, not gravity-aligned, rectified left and right image. Instead, we first have to project the line start and end points back to the unaligned images, select the maximum and minimum line start and end point and project the points back to compute a correct equally-sized patch for both lines.

Having computed the SAD for all potential matches, the line with the lowest SAD value in the right image is accepted as correct match for the line in the left image, if the SAD value is lower than a threshold.

We assume that the detected vertical lines are projections of vertical lines in the 3D scene to the image. Therefore, to compute the depth of the line, we just have to compute one disparity value for the whole line. However, as the epipolar constraints do not hold for the aligned image, we again project the lines into the unaligned images to compute the line disparity.

\subsection{Supporting Points}

When lines exist in the scene, they deliver a good texture for direct image alignment. However, also in man-made environments consisting mainly of lines, there are areas where no or just a few lines exist. Therefore we need a fall-back solution for the direct image alignment for images where not many or no lines can be detected. We selected to use patches around feature points in this case.

\subsubsection{Feature Point Detection}

If no sufficient area of the image is covered by detected lines, we detect point features in areas of the image which are not covered by line features. To determine the coverage of features over the image, the image is partitioned into a $5 \times 5$ grid of equally sized cells. Every cell which contains a line or parts of a line is marked as covered, all other cells are marked as uncovered. If less than 20 of the 25 cells are marked as covered, point detection is applied. However, as in cells marked as covered already information from lines exist, we just extract feature points in the uncovered cells. 
For every uncovered cell, we detect FAST corners (Rosten and Drummond, 2005) using an adaptive threshold. If the number of detected corners falls under 10, we decrease the corner threshold, while if the number of detected corners exceeds 20, we increase the threshold. Then, we store the determined thresholds for each cell for the next image to be processed.

\subsubsection{Feature Point Matching}

Having corresponding feature points in two images and a calibrated stereo setup, one can triangulate a 3D point. Therefore, we need to find points in the right image corresponding to points in the left image.

To find point matches, we proceed as follows: For every detected feature point in the left image, we search for detected feature points in the right image lying on the corresponding epipolar line. Therefore, the points have to be projected back into the original unaligned image, as the epipolar constraint does not hold anymore for the aligned images.

We search for points between a minimum and maximum disparity value to ignore too far and too near points. Having found some potential matches, we compare the point from the left image with the potential matching points in the right image by computing the SAD for a patch around the point. The corresponding point with the lowest SAD is detected as match, if the SAD value is under a fixed threshold.

\subsection{Pose Estimation by Direct Image Alignment}

Given 3D lines and points from the previous keyframe, our pose estimation algorithm estimates the pose by aligning the patches around the lines and points in the keyframe with the patches around the lines and points projected into the current frame (see Fig. 4). Alignment is done by non-linear least squares optimization on the intensity image.

\subsubsection{Definitions and Notation}

In this section, we state some definitions and notation conventions which are used in the following algorithm description.

In terms of notation, we denote matrices by bold capital letters (e.g., $T$ ) and vectors by bold lower-case letters (e.g., $\xi$ ).

A rigid body transformation $T \in S E(3)$ is defined as

$$
T=\left(\begin{array}{cc}
R & t \\
0 & 1
\end{array}\right) \text { with } R \in S O(3) \text { and } t \in \mathbb{R}^{3} .
$$

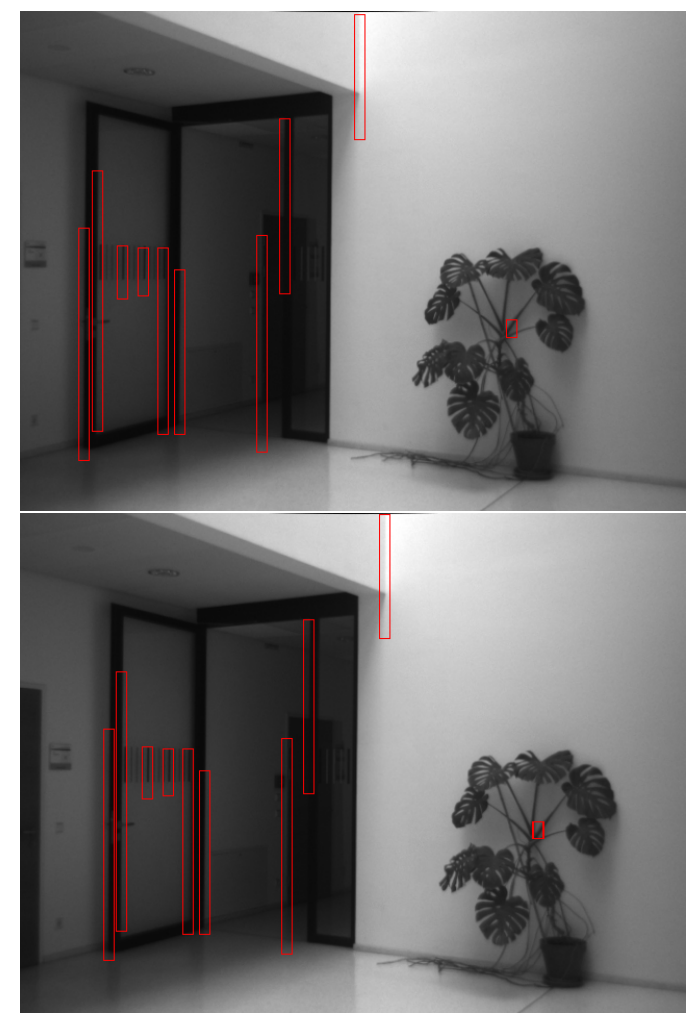

Figure 4: Direct image alignment using patches around lines. Left: Keyframe, where lines are detected and patches around lines (red boxes) are extracted. Right: Image to align. Red boxes are shifted according to the proposed new pose until a minimal photometric error is reached.

Having a transformation $T_{0, i}$ which transforms the origin 0 into camera $i$ and maps points from the coordinate frame of $i$ to the origin 0 , the same transformation can be assembled having a transformation $T_{0, i-1}$ from a previous camera $i-1$ and a transformation $T_{i-1, i}$ describing the transformation from the camera $i-1$ to camera $i$ :

$$
T_{0, i}=T_{0, i-1} \cdot T_{i-1, i}
$$

For the optimization, we need a minimal description of transformations. Therefore, we use the Lie algebra $\mathfrak{s e}(3)$, which is the tangent space of the matrix group $S E(3)$ at the identity, and its elements are denoted as twist coordinates

$$
\xi=\left(\begin{array}{l}
\omega \\
v
\end{array}\right) \in \mathbb{R}^{6},
$$

where $\omega$ defines the rotation and $v$ defines the translation. As stated in (Ma et al., 2003), twist coordinates can be mapped onto $S E(3)$ by using the exponential map:

$$
T(\xi)=\exp (\xi) \text {. }
$$




\subsubsection{Non-Linear Least Squares Optimization on Image Intensities}

To estimate the 6 DoF pose of a new image frame given the image patches around lines and points from the previous keyframe, we minimize the photometric error for the twist $\xi$ by applying non-linear least squares optimization. We keep the 3D lines and points fixed in the optimization step, as we assume to have already accurately triangulated $3 \mathrm{D}$ information from using the calibrated stereo setup.

The quadratic photometric error is defined as follows:

$E(\xi)=\sum_{i=1}^{n}\left(I_{k f}\left(p_{i}\right)-I\left(\pi\left(p_{i}, d_{k f}\left(p_{i}\right), \xi\right)\right)\right)^{2}=\sum_{i=1}^{n} r_{i}^{2}(\xi)$,

where $I_{k f}\left(p_{i}\right)$ is the intensity value of position $p_{i}$ in the keyframe to align to, $\pi\left(p_{i}, d_{k f}\left(p_{i}\right), \xi\right)$ denotes the warping function which warps point $p_{i}$ of the keyframe into the current image and $I$ is the current intensity image. This function computes all $n$ residual values $r_{i}(\xi)$ for every pixel $i$.

In order to decrease the influence of outliers, i.e. patches with very high residual values, we use a Cauchy Loss function to down-weight high residuals.

For performance reasons, we do not warp all pixels of the patches in image $I_{k f}$ to image $I$ but just the central top and bottom points of the patches. For residual computation we then compare patches around the warped points in $I$ with the patches from $I_{k f}$. This simplification is valid for small patches and small motion between frames and also similarly used by others (e.g. (Forster et al., 2014)).

For all of the image patches, we seek to find a solution for $\xi$ which minimizes the total squared photometric error

$$
\xi=\arg \min _{\xi} \frac{1}{2} E(\xi) .
$$

The minimum value of $\xi$ occurs when the gradient is zero:

$$
\frac{\partial E}{\partial \xi_{j}}=2 \sum_{i=1}^{n} r_{i} \frac{\partial r_{i}}{\partial \xi_{j}}=0,(j=1, \ldots, 6)
$$

As Eq. 6 is non-linear, we iteratively solve the problem. In every iteration, we linearize around the current state in order to determine a correction $\Delta \xi$ to the vector $\xi$ :

$$
E(\xi+\Delta \xi) \approx E(\xi)+J(\xi) \Delta \xi,
$$

where $J(\xi)$ is the Jacobian matrix of $E(\xi)$. It has a size of $n \times 6$ with $n$ as the number of residual values and is given by:

$$
J_{i, j}(\xi)=\frac{\partial E_{i}(\xi)}{\partial \xi_{j}}
$$

Using this linearization, the problem can be stated as a linear least squares problem:

$$
\Delta \xi=\arg \min _{\Delta \xi} \frac{1}{2}(J(\xi) \Delta \xi+E(\xi)) .
$$

In our approach, we use the Levenberg-Marquardt algorithm (Levenberg, 1944) (Marquardt, 1963) to solve the linear least squares problem stated in Eq. 10.

By rearrangement and plugging Eq. 8 and Eq. 9 into Eq. 7, we obtain the following normal equations:

$$
J(\xi)^{T} J(\xi) \Delta \xi=-J(\xi)^{T} E(\xi) .
$$

We solve this equation system by using QR decomposition.

Having computed the twist $\xi$, we can use Eq. 2 and Eq. 4 to compute an updated camera pose

$$
T_{0, i}=T_{0, k f} \cdot T(\xi),
$$

where $T_{0, i}$ is the current pose, $T_{0, k f}$ is the pose of the last keyframe and $T(\xi)$ is the movement from the last keyframe to the current frame.

If the IMU measurements used for the gravity alignment of the images were exact all the time, the problem would be reducible to $4 \mathrm{DoF}$, as then roll and pitch would be constantly 0 . However, as the IMU measurements contain errors, also roll and pitch can vary. Therefore, we don't fix roll and pitch but just allow small rotations.

Even though patches around lines do not contain a lot horizontal structure, the horizontal structure contained in the patches is enough to align the images correctly also in vertical direction.

\subsection{Keyframe Selection and Map Extension}

After a certain amount of motion, a new keyframe has to be selected and consecutively new lines and possibly points have to be matched and triangulated.

We use a simple measure for keyframe selection, which proved to work well in our experiments: After the pose estimation step, we compute the average photometric error per pixel. If this error exceeds a certain threshold, the current frame is selected as new keyframe. Additionally, if the translation and rotation w.r.t. the last keyframe exceeds a threshold, the current frame is selected as new keyframe.

For every keyframe, new lines and points are detected. However, before triangulating new lines or points, the already triangulated lines and points of the previous keyframe get projected into the current keyframe. If they are near a detected line/point in the current keyframe, the already computed depth value gets assigned to the lines/points in the current 
keyframe. All remaining detected lines get matched and triangulates as described in Sec. 3.2 and additional points are used if necessary as described in Sec. 3.3.

\section{EXPERIMENTS AND RESULTS}

In this section, we first describe our implementation used for the experiments in more detail and then evaluate our method on our own challenging indoor dataset acquired with a flying MAV, on the publicly available Rawseeds dataset (Bonarini et al., 2006) (Ceriani et al., 2009) and on a subset of the KITTI dataset (Geiger et al., 2012). We show that our approach outperforms state-of-the-art methods on the first two challenging indoor datasets and delivers comparable results on the last dataset.

\subsection{Implementation Details}

We implemented our algorithm in $\mathrm{C}++$ using OpenCV as image processing library and Ceres Solver (Agarwal et al., ) for the optimization tasks.

In our evaluation, we used the images in full size, as downsampled images with a small stereo setup baseline significantly decreased the quality of the triangulated $3 \mathrm{D}$ information.

For line detection, we set the gradient histogram bin size to 20 which has shown to deliver good detection results. In the non-maximum suppression step, we take into account lines with a distance not farther than $1.5 \%$ of the image width. For the matching, a line is matched with another one if their gradient histogram bins do not differ by more than 2 . Then, a match is accepted if the photometric error of the patch around the line has a smaller average photometric error per pixel than 15 .

In the optimization step, we initialize the pose estimation of a new frame with the pose of the last frame. In order to avoid random jumps and rotations when too few or erroneous measurements are used in the optimization, we set upper and lower bounds for maximum rotational and translational movements w.r.t. the last keyframe: The maximum translational movement is set to $1 \mathrm{~m}$ and the maximum rotational movement is set $35 \mathrm{deg}$. These thresholds multiplied by 0.9 are also used for keyframe selection. In patch alignment, bilinear image interpolation is used to determine the interpolated image intensity and gradient values. For lines, we use a patch width of 21 pixels and height according to the line length. For points we use a patch size of $21 \times 21$ pixels.

\subsection{Evaluation Setup}

We evaluated our algorithm on a desktop PC which is an Intel Core i7-4820K CPU having 4 cores with $3.7 \mathrm{GHz}$, up to 8 threads and $16 \mathrm{~GB}$ of RAM. However, our algorithm just used 4 threads for computation.

We compared our approach against Libviso (Geiger et al., 2011), which is a sparse feature-point based stereo visual odometry approach. We used it with subpixel refinement, set bucket height and width to $100 \mathrm{px}$, maximum features per bucket to 10 and match radius to 50, as these settings have shown to deliver good results on our MAV dataset and the Rawseeds dataset. For one test sequence, we also compared with LSD-Slam (Engel et al., 2014), which is a direct monocular SLAM approach. However, we didn't use LSD-SLAM in the other experiments, as the sequences are not suited for a monocular approach, which cannot handle pure rotations and has problems with pure forward motion. We disabled loop closure and global map optimization in LSD-SLAM by setting the doSLAM option to false, as these trajectory improvement techniques are also not used in our approach.

\subsection{Own Test Sequences}

To evaluate the performance of our algorithm in challenging indoor environments where just few texture is available, we captured an image sequence by flying with an MAV in a room containing few texture elements. As capturing system, we used an Asctec Pelican equipped with two forward and slightly downwards looking Matrix Vision BlueFoxMLC202b cameras with a baseline of approximately $13.5 \mathrm{~cm}$ (see Fig. 5). In this setup, each camera has a horizontal field of view of $81.2 \mathrm{deg}$ and acquires images with 20 frames per second (FPS) at a resolution of $640 \times 480$ pixels. In order to capture synchronized images and IMU measurements, we externally trigger the cameras with the Asctec Autopilot. Simultaneously to the trigger signal, the Asctec Autopilot captures a timestamped IMU measurement. Both, the images and the IMU measurements are then stored on the Odroid XU3 Lite computation board onboard the MAV. We calibrated both the stereo camera setup and the rotation from IMU to camera with the Kalibr toolbox (Furgale et al., 2013).

The sequence is captured while flying with the MAV in front of a wall in a broad hallway. The trajectory is similar to a rectangle, while both cameras are looking in the direction of the wall all the time. The start and end points are nearly identical, therefore the 


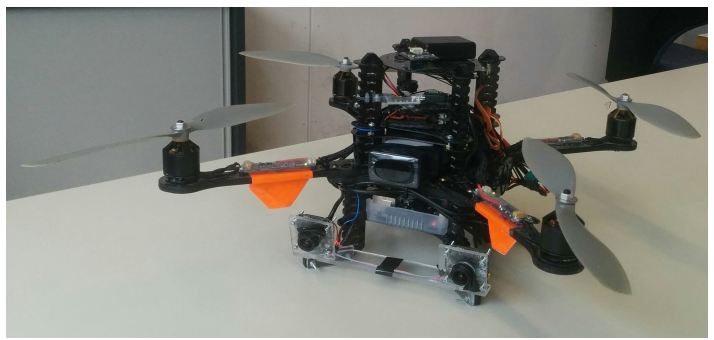

Figure 5: The Asctec Pelican used to acquire our own test dataset. It is equipped with an IMU (included in the autopilot), a stereo camera and an Odroid XU3 Lite processing board.
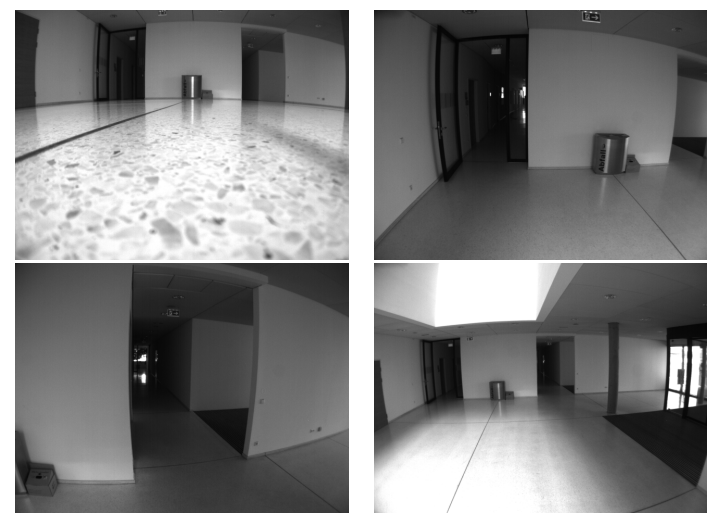

Figure 6: Images of the sequence acquired with the flying MAV. As can be seen, the scene mostly consists of white walls with little texture.

absolute trajectory error can be observed. This scenario is similar to an augmented reality application, where, for example, an MAV equipped with a laser projector should project something onto the wall.

As can be seen in the input images in Fig. 6, the scene mostly consists of white, untextured walls. Only few structure elements are visible (doors, recycle bin).

We compare this sequence with Libviso (Geiger et al., 2011) and LSD-SLAM (Engel et al., 2014). As LSD-SLAM is a monocular approach and therefore does not provide a metric scale, we manually aligned the trajectory scale with the stereo approaches. However, generally this trajectory should be well suited for a monocular approach, as there are no pure rotations and no forward movement in the initialization phase.

The computed trajectories are plotted in Fig. 7. In comparison to Libviso (blue), our approach (red) does not have a big drift. The start/end point difference of our approach is approximately $2.5 \mathrm{~m}$ while Libviso has a difference of approximately $7 \mathrm{~m}$. Libviso has already a big translational drift when the MAV is standing still on the floor at the beginning of the trajectory. While flying, Libviso accumulates a big rotational drift, which leads to big changes in the z- dimension. Contrary, LSD-SLAM estimates parts of the trajectory quite well. However, some random pose jumps happen in the estimation, which yields to a trajectory which is partly far away from the correct solution. In total, our approach performs best compared to the others, as it does not have a big drift and no big outliers.

\subsection{Rawseeds}

The Rawseeds datasets (Bonarini et al., 2006) (Ceriani et al., 2009) are publicly available indoor and outdoor datasets captured with a ground robot equipped with multiple sensors. Additionally, ground truth data was captured for some parts of the trajectories with an external tracking system.

In our experiments, we chose to use the Bicocca_2009-02-27a dataset, which is an indoor dataset captured in a static environment with mixed natural and artificial lightning. Note that this is a really largescale dataset, as the total trajectory length is 967.18 $\mathrm{m}$. We used the left and right images of the trinocular forward-looking camera for our experiments, which have a resolution of 640x480 pixels each, a baseline of $18 \mathrm{~cm}$ and were captured with 15 FPS. As the images were acquired roughly aligned with the gravity direction, no IMU measurements are needed for our algorithm. Example input images can be seen in Fig. 8.

As the ground truth computed with an external tracking system does not cover the whole trajectory, we use the (publicly available) extended ground truth in our experiments. This ground truth also includes trajectory parts estimated with data of an onboard laser scanner.

In the experiments for this dataset, we compare our approach with Libviso. In Tab. 1 one can see the relative pose errors as proposed in (Sturm et al., 2012). Our approach gives better results for all computed relative pose errors. However, due to the difficulty of the sequence, which also contains images with no texture at all (see Fig. 8), the error is also relatively high when using our algorithm. Additionally, the framerate should be higher for our approach. As this dataset is just captured with 15 FPS, sometimes the scene just moves too fast in front of the camera and the camera pose cannot be tracked exactly. However, in the parts of the sequences where there exist some vertical line structure (e.g. doors, windows or shelves), our approach works better and therefore the errors are lower. 



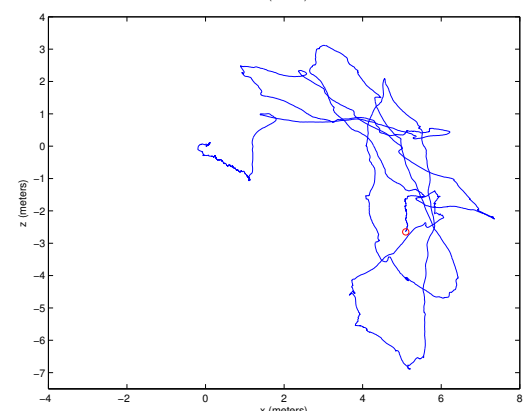

Libviso



Our Approach



LSD-SLAM

Figure 7: Estimated trajectories of Libviso (left, blue), our approach (middle, red) and LSD-SLAM (right, green) of the test sequence captured with an MAV. Top row: $\mathrm{x}$ - and y-axes (top view). Bottom row: $\mathrm{x}$ - and z-axes (side view). The start point is at $(0,0,0)$ and the trajectory end point, which should be near the start point is indicated with a circle. Even though our approach (red) has a slight drift (start point is not identical with end point), Libviso (blue) has a much higher rotational drift. LSD-SLAM works well for some parts of the sequence. However, occasionally it does some random jumps and moves far away from the desired trajectory.
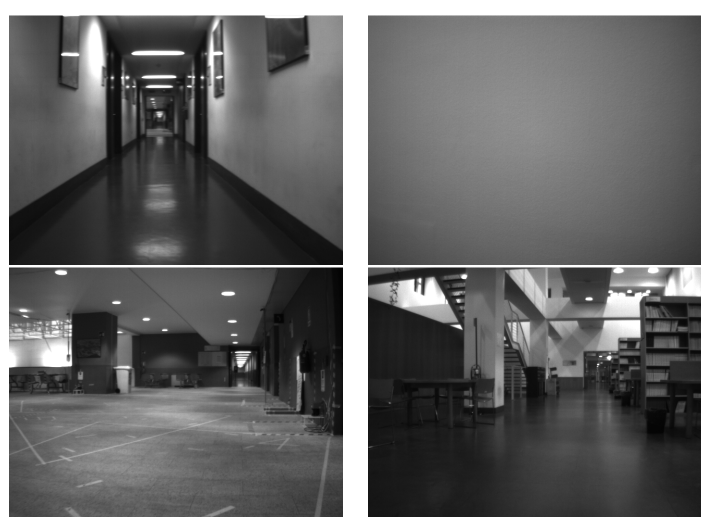

Figure 8: Images of the Rawseeds Bicocca_2009-02-27a sequence. Top left: A big part of the images look similar to this, where the robot moves through a narrow corridor. Top right: At some corridor intersections, where nearly pure rotations are performed, just the white wall is captured with the cameras, which is extremely difficult for a VO algorithm Bottom row: Also parts with better input data for VO exist, e.g., wider hallways and a library.

\section{$4.5 \quad$ KITTI}

The KITTI dataset (Geiger et al., 2012) is currently a popular dataset for visual odometry evaluation. It is
Table 1: Relative translational and rotational error of the Rawseeds sequence as defined in (Sturm et al., 2012) per seconds of movement of our approach and Libviso. In all metrics, our approach performs better.

\begin{tabular}{|l||l|l|}
\hline & Our Approach & Libviso \\
\hline Transl. RMSE (m/s) & $\mathbf{0 . 6 6 5}$ & 0.753 \\
\hline Transl. Median (m/s) & $\mathbf{0 . 5 7 7}$ & 0.68 \\
\hline Rot. RMSE (deg/s) & $\mathbf{1 0 . 8 9 2}$ & 12.229 \\
\hline Rot. Median (deg/s) & $\mathbf{0 . 0 1 8}$ & 0.033 \\
\hline
\end{tabular}

acquired with a stereo camera mounted on a car which drives on public streets, mostly through cities. We compare our algorithm against Libviso (Geiger et al., 2011) on a subset of the dataset (sequence 07).

The images have a resolution of $1241 \times 376$ pixels and a framerate of 10 FPS. As the images are roughly aligned with the gravity direction, there is no need to incorporate IMU measurements. Due to the low capture framerate and the relatively fast movement of the car, the image content can change quite rapidly. This is a problem for direct VO methods which deliver good results when having small inter-frame motions. Additionally, it contains enough texture for featurebased methods to work properly. However, we want to show that our approach works also well on datasets 


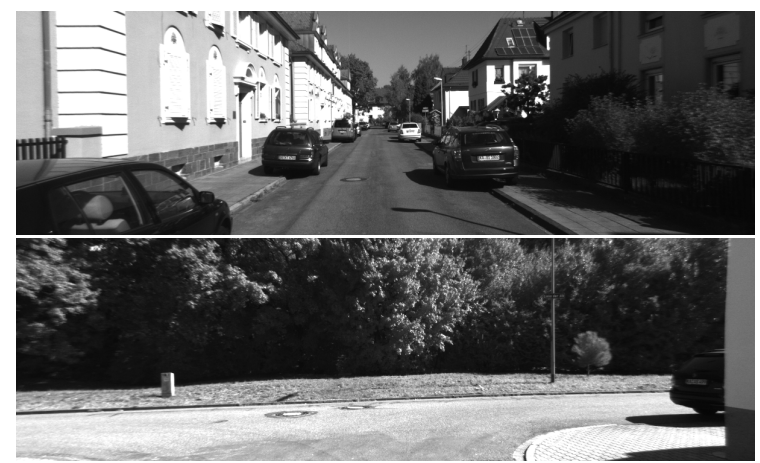

Figure 9: Images of the KITTI visual odometry dataset (sequence 07 ). In this sequence, there are some lines detectable in some parts (e.g. in top image). In these parts, the detected lines are beneficial for the VO algorithm. However, in some images there exist no lines (e.g. image bottom) and our algorithm also depends on the detected feature points.

Table 2: Relative translational and rotational error of the KITTI sequence 07 computed with the KITTI vision benchmark suite (Geiger et al., 2012) as mean over all possible subsequences of length $(100, \ldots, 800)$ meters.

\begin{tabular}{|l||l|l|}
\hline & Our Approach & Libviso \\
\hline Transl. error $(\%)$ & 8.0558 & $\mathbf{2 . 3 6 7 6}$ \\
\hline Rot. error $(\mathrm{deg} / \mathrm{m})$ & 0.000744 & $\mathbf{0 . 0 0 0 3 2 1}$ \\
\hline
\end{tabular}

for which it is not specifically designed for.

To overcome the low framerate, we had to change the patch size of the patches around lines and points used in the optimization from 21 to 31 . Additionally, we had to increase the maximal translational movement for the optimization step. Using this settings we get results comparable to state-of-the-art methods with the drawback of higher runtimes compared to the other datasets used in our evaluation. Additionally, as more reliable points can be detected in this scene, we decreased the line matching threshold so that more points are used.

Also for Libviso, we changed the parameter settings: We used it with default settings and subpixel refinement.

In Fig. 10 the computed trajectories of Libviso and our approach are plotted. As one can see, the absolute trajectory error is slightly worse in our approach. Also the rotational and translational errors as defined in the KITTI vision benchmark suite are worse than Libviso (see Tab. 2). However, our algorithm still works acceptable on a dataset like this one for which it is not designed for.

\subsection{Runtime}

The mean computation time per frame for the first two datasets is $49.3 \mathrm{~ms}$, which is a framerate of 20.3

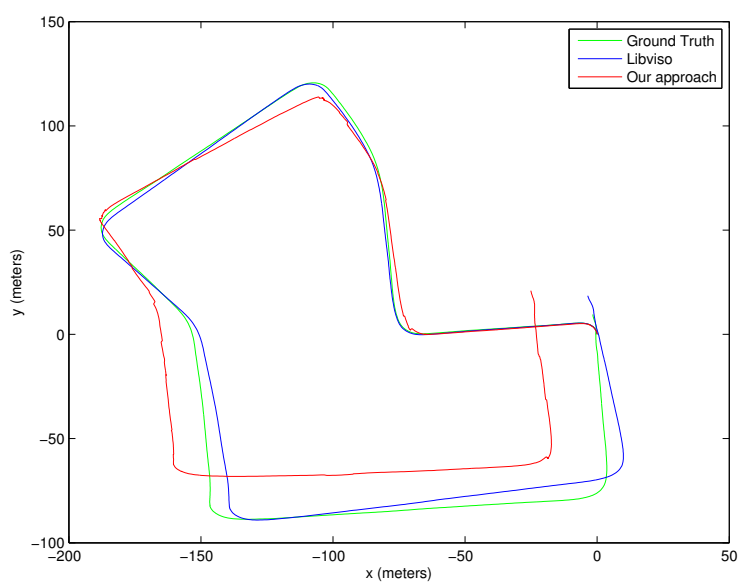

Figure 10: Evolving trajectory of the KITTI sequence. One can observe that our approach (red) performs comparable to Libviso (blue).

FPS. With this computation speed, both the Rawseeds dataset and our own MAV dataset can be processed in real-time. Due to changed parameters for the KITTI dataset, the computation speed for the KITTI dataset is much slower and needs approximately 3 times the processing time of the other datasets.

Most of the processing time is needed for the pose estimation step, which could be optimized by a multi-scale approach, using SIMD instructions (SSE, NEON) or writing an own optimizer to overcome the overhead of Ceres.

\section{CONCLUSION}

We have presented a direct stereo visual odometry method, which detects and matches lines at every keyframe using our novel fast line detection algorithm and estimates the pose of consecutive frames by direct pose estimation methods using patches around vertical lines. These patches have proven to be a good selection for indoor environments, which are poorly textured, but where a lot of vertical structure exists. In our experiments, we have shown that our algorithm delivers better results than state-of-the-art methods in challenging indoor environments and comparable results in well textured outdoor environments. As our implementation runs in real-time, it is suitable for various robotics and augmented reality applications. Future work will include subpixel refinement of the line detection step, global map optimization to minimize drift errors and to evolve it to a complete SLAM system, a multi-scale approach for the pose estimation step to deliver better accuracy for large interframe movements and improve computation speed, 
and further speed improvements (e.g., using SSE and NEON instructions) to make it real-time capable also on small robotic platforms.

\section{ACKNOWLEDGEMENTS}

This project has been supported by the Austrian Science Fund (FWF) in the project V-MAV (I-1537).

\section{REFERENCES}

Agarwal, S., Mierle, K., and Others. Ceres solver. http://ceres-solver.org.

Bonarini, A., Burgard, W., Fontana, G., Matteucci, M., Sorrenti, D. G., and Tardos, J. D. (2006). Rawseeds: Robotics advancement through webpublishing of sensorial and elaborated extensive data sets. In International Conference on Intelligent Robots and Systems.

Ceriani, S., Fontana, G., Giusti, A., Marzorati, D., Matteucci, M., Migliore, D., Rizzi, D., Sorrenti, D. G., and Taddei, P. (2009). Rawseeds ground truth collection systems for indoor selflocalization and mapping. Autonomous Robots, 27(4):353-371.

Cortinovis, A. (2010). Pixhawk - attitude and position estimation from vision and imu measurements for quadrotor control. Technical report, Computer Vision and Geometry Lab, Swiss Federal Institute of Technology (ETH) Zurich.

Elqursh, A. and Elgammal, A. M. (2011). Line-based relative pose estimation. In Proceedings IEEE Conference Computer Vision and Pattern Recognition, pages 3049-3056. IEEE Computer Society.

Engel, J., Schöps, T., and Cremers, D. (2014). Lsdslam: Large-scale direct monocular slam. In Proceedings European Conference on Computer Vision.

Engel, J., Stückler, J., and Cremers, D. (2015). Largescale direct slam with stereo cameras. In International Conference on Intelligent Robots and Systems.

Forster, C., Pizzoli, M., and Scaramuzza, D. (2014). Svo: Fast semi-direct monocular visual odometry. In International Conference on Robotics and Automation.

Furgale, P., Rehder, J., and Siegwart, R. (2013). Unified temporal and spatial calibration for multisensor systems. In International Conference on Intelligent Robots and Systems.
Geiger, A., Lenz, P., and Urtasun, R. (2012). Are we ready for autonomous driving? the kitti vision benchmark suite. In Proceedings IEEE Conference Computer Vision and Pattern Recognition.

Geiger, A., Ziegler, J., and Stiller, C. (2011). Stereoscan: Dense $3 \mathrm{~d}$ reconstruction in real-time. In IEEE Intelligent Vehicles Symposion.

Grompone, R., Jakubowicz, J., Morel, J. M., and Randall, G. (2010). Lsd: A fast line segment detector with a false detection control. IEEE Transactions on Pattern Analysis and Machine Intelligence, 32(4):722-732.

Hofer, M., Maurer, M., and Bischof, H. (2014). Improving sparse $3 \mathrm{~d}$ models for man-made environments using line-based $3 \mathrm{~d}$ reconstruction. In International Conference on $3 D$ Vision.

Klein, G. and Murray, D. (2007). Parallel tracking and mapping for small ar workspaces. In Proceedings International Symposium on Mixed and Augmented Reality.

Levenberg, K. (1944). A method for the solution of certain non-linear problems in least squares. In Quarterly of Applied Mathematics 2, pages 164168.

Ma, Y., Soatto, S., Kosecka, J., and Sastry, S. S. (2003). An Invitation to 3-D Vision: From Images to Geometric Models. Springer Verlag.

Marquardt, D. (1963). An algorithm for least-squares estimation of nonlinear parameters. In SIAM Journal on Applied Mathematics 11(2), pages 431-441.

Mei, C., Sibley, G., Cummins, M., Newman, P., and Reid, I. (2010). Rslam: A system for large-scale mapping in constant-time using stereo. In International Journal of Computer Vision.

Newcombe, R. A., Lovegrove, S. J., and Davison, A. J. (2011). Dtam: Dense tracking and mapping in real-time. In Proceedings International Conference on Computer Vision, pages 2320-2327.

Rosten, E. and Drummond, T. (2005). Fusing points and lines for high performance tracking. In Proceedings International Conference on Computer Vision.

Sturm, J., Engelhard, N., Endres, F., Burgard, W., and Cremers, D. (2012). A benchmark for the evaluation of RGB-D SLAM systems. In International Conference on Intelligent Robots and Systems, pages 573-580.

Weiss, S., Achtelik, M. W., Lynen, S., Achtelik, M. C., Kneip, L., Chli, M., and Siegwart, R. (2013). Monocular vision for long-term micro aerial vehicle state estimation: A compendium. Journal of Field Robotics, 30(5):803-831. 